\title{
Transmitting CMUT Arrays without a DC Bias
}

\author{
Kerem Enhos \\ Department of Electrical \\ and Electronics \\ Engineering, Bilkent \\ University \\ Ankara, Turkey \\ enhos@ee.bilkent.edu.tr
}

\author{
A. Sinan Tasdelen \\ Bilkent University \\ Acoustics and Underwater \\ Technologies Research \\ Center, Bilkent University \\ Ankara, Turkey \\ sinan@ee.bilkent.edu.tr
}

\author{
Mehmet Yilmaz \\ UNAM-National \\ Nanotechnology Research \\ Center, Institute of \\ Material Science and \\ Nanotechnology, Bilkent \\ University \\ Ankara, Turkey \\ mehmetyilmaz@unam.bilk \\ ent.edu.tr
}

\author{
Abdullah Atalar \\ Department of Electrical \\ and Electronics \\ Engineering, Bilkent \\ University \\ Ankara, Turkey \\ aatalar@bilkent.edu.tr
}

\author{
Hayrettin Koymen \\ Department of Electrical \\ and Electronics \\ Engineering, Bilkent \\ University \\ Ankara, Turkey \\ koymen@ee.bilkent.edu.tr
}

\begin{abstract}
This study focuses on design, simulation, fabrication and measurement of transmitting CMUT arrays with unbiased mode of operation. We presented a design procedure considering the radiation pattern, Rayleigh distance and the parameters of the large signal equivalent circuit, which can be applied for different operation frequencies and applications. Large signal equivalent circuit model is used for lumped-element simulations. Harmonic balance and transient analyses are carried out with Gaussian enveloped tone burst, sinusoidal and pulse width modulation (PWM) signals with different duty cycles. Outputs of these simulations are fed in beamforming toolboxes for further verification. According to the design specifications transmitting CMUT arrays are fabricated. Corresponding experimental impedance measurements are conducted.
\end{abstract}

\section{Keywords-CMUT, unbiased, array, half frequency, transmit}

\section{INTRODUCTION}

For designing and simulating arrays that consist of large number of CMUT cells, lumped element modeling is a feasible and computationally low cost method. By defining mutual radiation impedance matrix, each CMUT cell's behavior and interaction between CMUT cells can be predicted. In our previous studies, simulation of uncollapsed CMUT arrays has been verified with lumped element modeling [1].

Previously, unbiased mode of operation for airborne CMUT cells has been presented [2]. A large plate swing can be obtained at low excitation voltages using the entire remaining gap. However, in biased operation, entire gap cannot be used due to collapsing. Thus, using the entire gap for plate motion gathers a huge advantage for transmitting CMUTs, which results in higher acoustical power output for lower excitation voltages. The minimum voltage level that provides plate swing over the entire gap is called minimum voltage mode $(M V M)$. Also the absence of DC bias voltage prevents charging of the CMUTs, which is a crucial advantage of this mode.

In unbiased mode, the resulting acoustical output signal is twice the frequency of sinusoidal electrical input signal. Force term in the acoustical output depends on the square of the input voltage as given in (1) [2]. With this feature, higher resolution transmitted signal waveform definition can be obtained when pulse width modulation (PWM) is employed for high frequency signals. Coded signals can be transmitted with PWM, for higher signal-to-noise ratio (SNR) ultrasonic images and beamforming [3].

$$
f_{R}(t)=\sqrt{5} \frac{C_{0} V^{2}(t)}{2 t_{g e}} g^{\prime}\left(\frac{x_{P}(t)}{t_{g e}}\right)
$$

In the design process of CMUTs, large-signal equivalent circuit model is used [4]. In order to have volumetric transmission, a $16 \times 16 \quad(256$ elements $)$ phased array configuration is chosen. Considering the radiation pattern, Rayleigh distance and the parameters of the lumped-element model, a design procedure is constructed. This procedure is applied for designing two arrays, with resonance frequencies at 7.5 $\mathrm{MHz}$ and at $18.5 \mathrm{MHz}$. Fabrication limitations and available resources are also considered during the design process as constraints. According to the determined design values simulations are completed with conventional lumped element simulation software. Outputs of the transient analysis are fed into a beamforming toolbox in order to resolve the problems associated with beamforming in this mode of operation. Thereafter, CMUT arrays are fabricated with anodic wafer bonding, in order to verify the simulation and experimental results.

\section{DESINGNING UNBIASED CMUT ARRAYS}

After determining the operation frequencies, design procedure is started with designating the radius of single cell, $a$, and the pitch of the elements in the array, $d$. These values are selected by considering the Rayleigh distance and the minimum sidelobe level of the array. According to application, selection criteria can be modified, whereas in this case biomedical application is aimed. Thus, maximum Rayleigh distance for higher beamformable region and minimum sidelobe level is desired. For obtaining the sidelobe level, 2D array factor [5] is multiplied with the radiation pattern for clamped disk for single element by using third order Bessel function as described in [6]. Both Rayleigh distance [5] and radiation pattern are dependent on the radius and pitch values. By this way, an optimal radius and pitch value can be obtained by using predetermined constraints for these values.

After determining the radius of the elements, the plate thickness, $t_{m}$, can be obtained by using the resonance equation in [4]

$$
\omega_{m}=\frac{1}{\sqrt{L_{A m} C_{A m}}}=\frac{t_{m}}{a^{2}} \sqrt{\frac{80}{9} \frac{Y_{0}}{\rho_{m}\left(1-\sigma^{2}\right)}}
$$

where density, $\rho$, is $2370 \mathrm{~kg} / \mathrm{m}^{3}$, Poisson ratio, $\sigma$, is 0.17 , and the Young's modulus, $Y_{0}$, is $149 \mathrm{GPa}$ for silicon in this work. The dynamic force acting on the membrane is the sum of the forces across the compliance and the mass. Hence the 
compliance $\left(C_{A m}\right)$ and the effective inductive loading $\left(L_{A m}\right)$ are in series and yields a resonance frequency, which is already determined for this design process.

Before approaching to determine the gap height, collapse voltage in vacuum, $V_{r}$, should be chosen. In this case, maximum input voltage is limited by the available ultrasound measurement system in our facility, which is $75 \mathrm{~V}_{\mathrm{p}}$. Thus, the collapse voltage in vacuum is chosen to be $100 \mathrm{~V}$ for safety purposes. Also, this voltage level helps us to determine the thickness of the insulating layer which is aluminum oxide in this work. With adding safety margin to the maximum applicable voltage level, dielectric breakdown voltage can be set and insulator thickness; $t_{i}$ can be determined. Finally, the effective gap height can be found by using (3) and afterward physical gap height can be obtained [4].

$$
V_{r}=8 \frac{t_{m}}{a^{2}} t_{g e}^{3 / 2} t_{m}^{1 / 2} \sqrt{\frac{Y_{0}}{27 \varepsilon_{0}\left(1-\sigma^{2}\right)}}
$$

According to all these calculated parameters, $F_{p b} / F_{p g}$, can also be calculated as given in [4]. If this value is relatively low, it means that the static depression is low and leaves more room for dynamic movement of the plate with the expense of lower bandwidth [2]. By using this value, a very good approximation for collapse voltage, $V_{c}$, for CMUTs can be obtained [4]. Since in unbiased mode, collapsing of CMUTs does not occur, instead $M V M$ voltage can be approximated by (4). If $M V M$ is a predetermined value, $V_{r}$, can be modified accordingly, which will iteratively affect $t_{g e}, F_{p b} / F_{p g}$ and $V_{c}$.

$$
M V M \approx V_{c} \times \sqrt{2}
$$

Finally, for both $7.5 \mathrm{MHz}$ and $18.5 \mathrm{MHz}$ operation frequencies, this design procedure is applied. However, for fabrication purposes, available resources and capabilities in fabrication are considered for $7.5 \mathrm{MHz}$ and some parameters had to be modified accordingly. Whereas, for $18.5 \mathrm{MHz}$ operation frequency design, exact calculated values according to the design procedure is given in Table 1.

TABLE I. DESIGN PARAMETERS FOR UNBIASED TRANSMITTER ARRAYS

\begin{tabular}{|c|c|c|c|}
\hline \multicolumn{2}{|c|}{} & CMUT-I & CMUT-II \\
\hline$f$ & Resonance frequency $(\mathrm{MHz})$ & 7.5 & 18.5 \\
\hline$a$ & Plate radius $(\mu \mathrm{m})$ & 80 & 32.2 \\
\hline$d$ & Element pitch $(\mu \mathrm{m})$ & 192 & 77.8 \\
\hline$S L L$ & Sidelobe level $(\mathrm{dB})$ & -17.4 & -22.6 \\
\hline$R_{0}$ & Rayleigh Distance $(\mathrm{mm})$ & 46.2 & 18.5 \\
\hline$t_{m}$ & Plate thickness $(\mu \mathrm{m})$ & 15 & 5 \\
\hline$t_{g e}$ & Effective gap height $(\mathrm{nm})$ & 171.2 & 101.8 \\
\hline$t_{i}$ & Insulator thickness $(\mathrm{nm})$ & 300 & 300 \\
\hline$t_{g}$ & Gap height $(\mathrm{nm})$ & 137.8 & 68.5 \\
\hline$F_{p b} / F_{p g}$ & Normalized exerted pressure & $0.01 @$ SAP & $0.01 @$ SAP \\
\hline$M V M$ & Minimum Voltage Mode $(\mathrm{V})$ & 182.8 & 100.0 \\
\hline
\end{tabular}

\section{ARRAY SIMULATIONS}

In this work, Advanced Design Systems (ADS) (Keysight Technologies, Santa Rosa, CA, USA) and MATLAB is used coherently and recursively. All of the output signals are obtained by ADS according to the designed transducer specifications and these output signals are used for beamforming and radiation pattern analysis in MATLAB. Also the delay calculations that are made in MATLAB are fed into ADS for beamforming simulations. In all simulations, electrical input frequency is given as half of the desired acoustic output frequency and as the input voltage only $75 \mathrm{~V}_{\mathrm{AC}}$ is applied unless otherwise stated. Lastly, only elements where major changes can be seen are presented, such as the corner $\left(1^{\text {st }}\right.$ element), middle $\left(120^{\text {th }}\right.$ element $)$ and side $\left(128^{\text {th }}\right.$ element $)$ elements. The differences are mostly caused by the mutual radiation impedance and effect of the rigid baffle around the elements.

For frequency domain analysis, nonlinear simulations are done by harmonic balance (HB) analysis due to unbiased operation. According to the results, $232.8 \mathrm{~dB}$ re $1 \mu \mathrm{Pa}$ pressure is obtained at the operation frequency of $7.5 \mathrm{MHz}$. Apart from the operation frequency, peak at $6.36 \mathrm{MHz}$ with $240.6 \mathrm{~dB}$ re $1 \mu \mathrm{Pa}$ is observed. However, due to the narrow bandwidth structure, this peak is not considered as the resonance frequency in this work. Instead this frequency is called as the peak frequency.

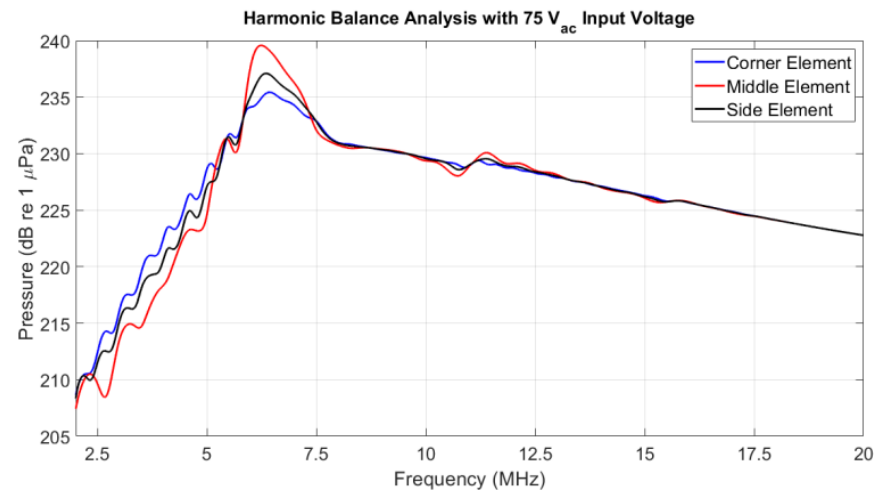

Fig. 1. Second harmonic results of harmonic balance analysis.

Comparison between unbiased and biased operation is also made. Average normalized membrane deflection caused by the $\mathrm{AC}$ voltage input in unbiased mode with $75 \mathrm{~V}_{\mathrm{AC}}$ is found to be 0.049 in transient analysis. The static depression in unbiased mode can only be done with ambient pressure, thus the biasing is equal to stress. Therefore, the same amount of deflection had to be maintained with DC bias for a fair comparison, and it is found to be $52.5 \mathrm{~V}_{\mathrm{DC}}$. For the AC input voltage, it is aimed to have the same output power in both operation modes, hence $\mathrm{AC}$ voltage set to $28.8 \mathrm{~V}_{\mathrm{AC}}$. The even harmonics of the electrical input's fundamental frequency in unbiased mode, which is $3.75 \mathrm{MHz}$, are compared with all the harmonics of the biased mode's input frequency, which is $7.5 \mathrm{MHz}$. According to obtained force values, total harmonic distortion (THD) is calculated in Table 2. Average of $12 \mathrm{~dB}$ difference is obtained between unbiased and biased mode of operation.

TABLE II. THD COMPARISON FOR UNBIASED AND BIASED MODE

\begin{tabular}{|c|c|c|}
\hline & Unbiased Mode (dB) & Biased Mode (dB) \\
\hline Corner Element & -35.42 & -25.65 \\
\hline Middle Element & -40.49 & -24.54 \\
\hline Side Element & -36.87 & -25.81 \\
\hline
\end{tabular}


Transient analyses are done with the same specifications. According to the simulations, at steady state $435.5 \mathrm{kPa}$ peak pressure is obtained from single CMUT element for transient sinusoidal waveform input.

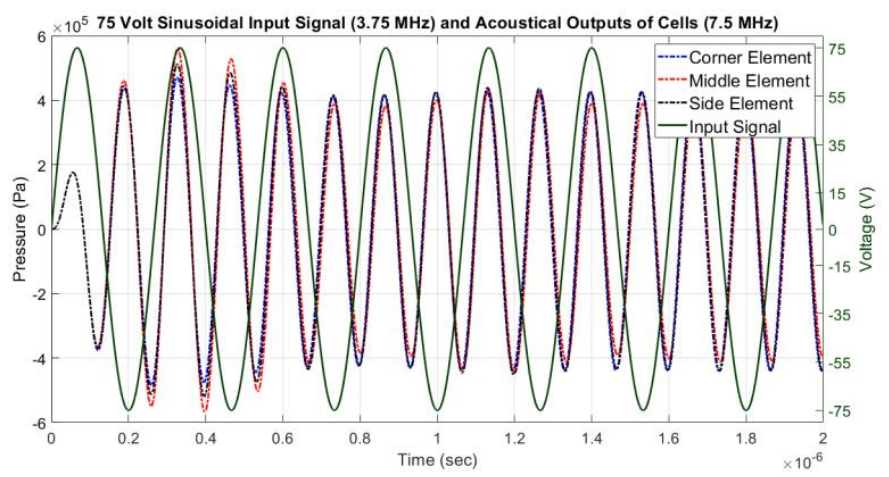

Fig. 2. Transient sinusoidal wave electrical input simulation.

Similarly, PWM signals are used as electrical input for the array. One cycle of PWM signal at $3.75 \mathrm{MHz}$ is generated and as output two cycles of $7.5 \mathrm{MHz}$ with $598.9 \mathrm{kPa}$ peak pressure is obtained.

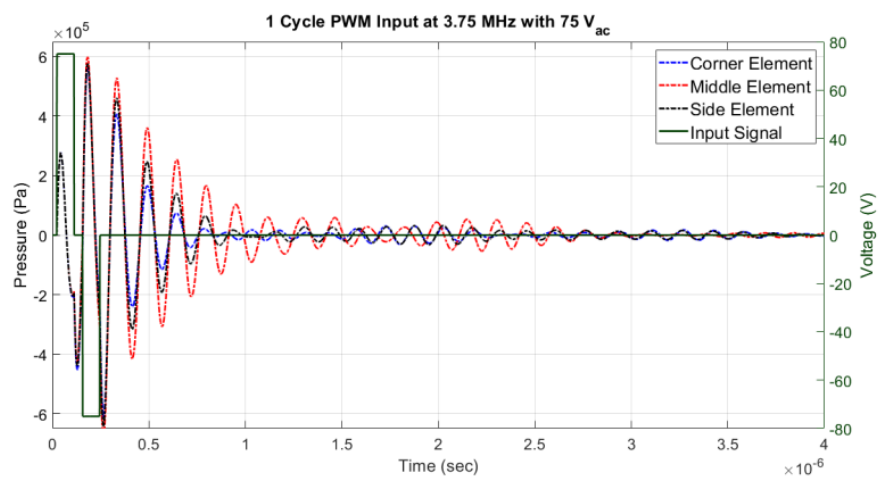

Fig. 3. One cycle PWM electrical input simulation.

Due to rapid peaks at the first cycle of transient analysis, applying Gaussian enveloped tone burst signal (GETB) is determined to be more appropriate for maintaining the plate swing that spans the entire gap. Maximum realizable normalized membrane displacement $\left(\mathrm{x}_{\mathrm{p}} / \mathrm{tg}_{\mathrm{ge}}\right)$ is calculated to be 0.8 . Thus, input voltage is set to maintain this level, which is equal to $M V M$. According to the simulations, $M V M$ is found to be $186 \mathrm{~V}_{\mathrm{AC}}$ with 5 cycle of GETB signal, which results in 2.764 MPa peak pressure for single CMUT cell.

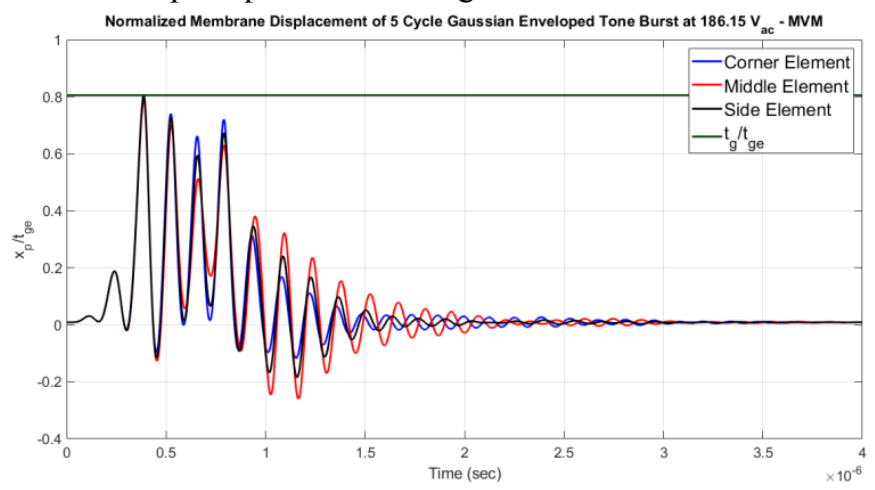

Fig. 4. $M V M$ is maintained at $186 \mathrm{~V}_{\mathrm{AC}}$ for Gaussian enveloped tone burst.
By using the transient analysis, power and intensity for different transmission waveforms with different duty cycles are calculated over the surface of the transducer as in Table 3.

TABLE III. POWER AND INTENSITY

\begin{tabular}{|c|c|c|c|c|}
\hline \multirow{2}{*}{} & \multicolumn{2}{|c|}{$\mathbf{7 5} \boldsymbol{V}_{\text {AC }}$ at $7.5 \mathbf{M H z}$} & \multicolumn{2}{c|}{$\boldsymbol{M V M}$} \\
\cline { 2 - 5 } & Power $(\boldsymbol{W})$ & $\begin{array}{c}\text { Intensity } \\
\left(\boldsymbol{W / c m}^{2}\right)\end{array}$ & Power $(\boldsymbol{W})$ & $\begin{array}{c}\text { Intensity } \\
\left(\boldsymbol{W} / \text { cm }^{2}\right)\end{array}$ \\
\hline Sine 0.5 Cycle & 0.0055 & 0.1113 & 0.2984 & 5.8994 \\
\hline Sine 1 Cycle & 0.0140 & 0.2952 & 0.3768 & 7.5010 \\
\hline Sine 4 Cycle & 0.0250 & 0.5567 & 0.4739 & 9.7263 \\
\hline GETB & 0.0146 & 0.3032 & 0.5856 & 10.8678 \\
\hline PWM 0.5 Cycle & 0.0085 & 0.1698 & 0.3000 & 5.5937 \\
\hline PWM 1 Cycle & 0.0213 & 0.4472 & 0.3947 & 7.3303 \\
\hline PWM 4 Cycle & 0.0390 & 0.7743 & 0.5201 & 9.0862 \\
\hline
\end{tabular}

Beamforming in this mode of operation is validated by comparing linearly increasing delay inputs with the lags between consecutive CMUT cells in a row of the array, which is obtained by their cross-correlation. According to this comparison, maximum difference is found to be $2 \mathrm{~ns}$, which is less than the time step size of our ultrasound measurement system. Thus, conventional beamforming is also applicable in unbiased mode of operation. After the validation, beam focusing is conducted with the output signals obtained by ADS and by feeding these signals into k-wave [7] for obtaining radiation patterns at different focus angles. K-wave is also used for obtaining field patterns as shown in Fig. 6.

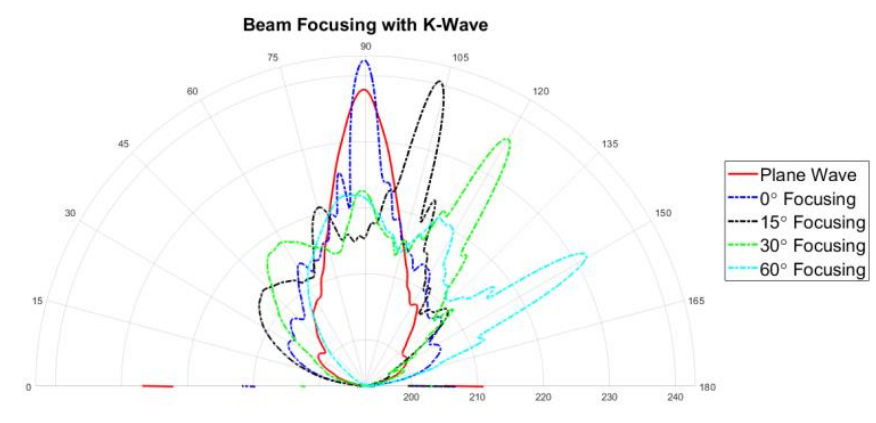

Fig. 5. Radiation patterns for focused transmission at different angles with one cycle PWM input.

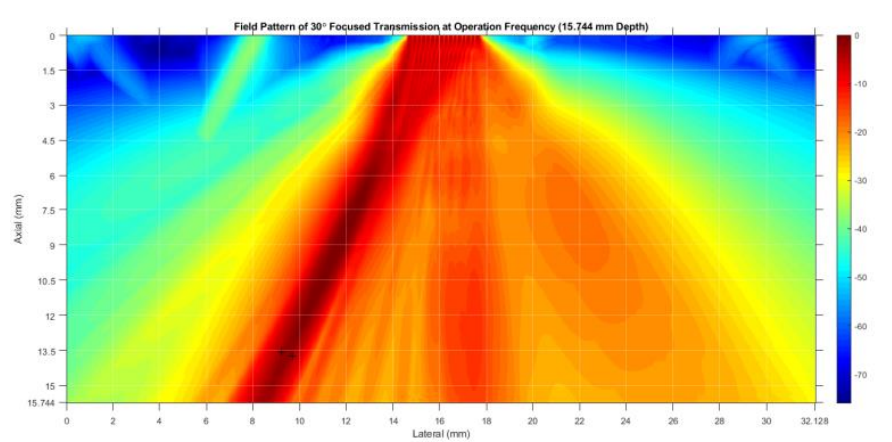

Fig. 6. Field pattern for $30^{\circ}$ focused transmission with one cycle PWM input over the elevation plane normal to the surface of the transducer. 


\section{FABRICATION}

Wafer scale batch compatible microfabrication is applied for fabrication of these MEMS devices. By considering the fabrication capabilities, minimum feature size of 3 um is used at first lithography step. All bottom electrical connections and CMUT cavities are formed by using the first lithography mask. Second lithography mask is used for opening up the electrical connection pad areas by etching alumina from the pad regions on Pyrex substrate. After anodic wafer bonding of SOI wafer with processed Pyrex substrate, the handle layer and buried oxide layer of the SOI wafer is reactive ion etched without using a mask. Next, the device layer of the SOI wafer is patterned to open the $\mathrm{Si}$ device layer from above the electrodes that already exist and does not have alumina due to the earlier second mask processing step in Pyrex substrate. The ground pads (top electrode) that are on Si device layer are deposited with physical vapor deposition using a shadow mask. Then, the wafer is diced into CMUT array chips as shown in Fig. 8a.

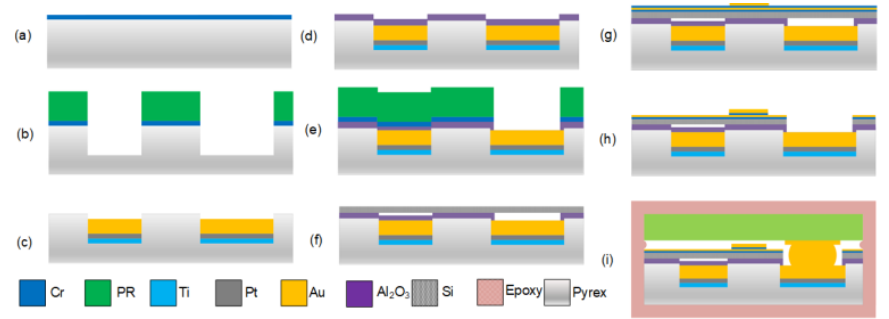

Fig. 7. Process flow of fabricated CMUT devices. Flip-chip bonding and sealing is done after fabrication (i).

Fabricated MEMS devices are flip-chip bonded to a designed PCB for measurement connections and sealed with flexible epoxy as shown in Fig. 8b. This PCB is integrated into a probe by using a $3 \mathrm{D}$ printed casing suitable for waterborne experiments.
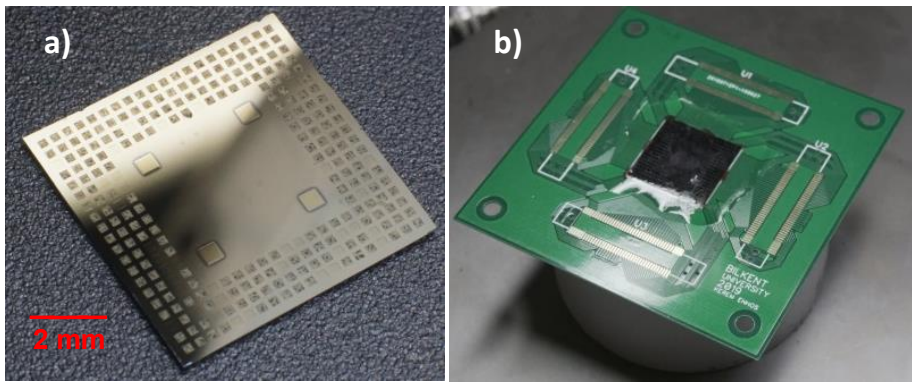

Fig. 8. Fabricated $16 \times 16$ unbiased phased array CMUT device.

\section{MEASUREMENTS}

According to the impedance simulations, it is observed that the conductance values are significantly low for accurate measurement. Thus, because of the high difference in the intrinsic acoustic impedance of silicon membrane and air, admittance measurements are conducted in air. According to measurements it is realized that the resonance frequencies are similar to the peak frequency in the HB analysis for waterborne. It is expected to have higher frequency resonances in airborne compared to waterborne measurements; however, in Fig. 9, the resonance frequency was measured to be at 6.9 $\mathrm{MHz}$, which is close to the peak frequency of the HB simulations, 6.36 MHz. This occurs because of driving only one element of the array while all the other cells in the array remain as dummy cells. Presence of these dummy cells matches with the vibrations in the medium and also the radiation of the single excited cell.

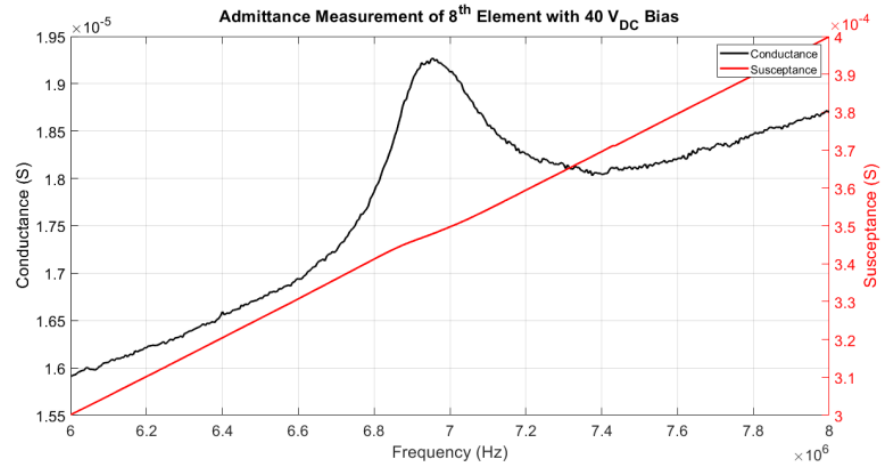

Fig. 9. Admittance measurement in air for single CMUT cell in the array.

\section{CONCLUSION}

This work focuses on the design procedure, simulation, fabrication and characterization of CMUT arrays with unbiased mode. Design procedure extended for array configuration by using the large signal equivalent circuit model and mutual radiation impedance matrix for computing this array with fairly large number of elements. Absence of DC bias prevents charging of CMUTs and coded signals can be transmitted with PWM signals generated at the frequency that is half of the desired acoustical output operation frequency for high SNR. A novel CMUT phased array working with unbiased mode, which can handle various waveform transmission schemes with half frequency electrical input drive, is presented.

\section{REFERENCES}

[1] H. K. Oguz, A. Atalar, and H. Koymen, "Equivalent circuit-based analysis of CMUT cell dynamics in arrays," IEEE Trans. Ultrason., Ferroelectr., Freq. Control, vol. 60, pp. 1016-1023, 2013.

[2] Aslı Ünlügedik, A. Sinan Taşdelen, Abdullah Atalar, and Hayrettin Köymen, "Designing Transmitting CMUT Cells for Airborne Applications," IEEE Trans. Ultrason., Ferroelectr., Freq. Control, vol. 61, no. 11, pp. 1899-1910, Nov. 2014.

[3] Yasin Kumru, Kerem Enhoș, and Hayrettin Köymen, "Pulse coding with complementary Golay sequences for signal to noise ratio improvement in ultrasound mammography," in Proceedings of Meetings on Acoustics, Acoustical Society of America, Victoria, Canada, 2018, pp. Volume 35, Issue 1, 020002.

[4] H. Köymen, A. Atalar, E. Aydoğdu, C. Kocabaş, H. K. Oğuz, S. Olçum, A. Özgürlük, and A. Ünlügedik, "An improved lumped element nonlinear circuit model for a circular CMUT cell," IEEE Trans. Ultrason. Ferroelectr. Freq. Control, vol. 59, no. 8, pp. 1791- 1799, Aug. 2012.

[5] David T. Blackstock, Fundamentals of Physical Acoustics. New York, United States of America: John Wiley \& Sons, 2000.

[6] D. Porter, "Self and Mutual-Radiation Impedance and Beam Patterns for Flexural Disks in a Rigid Plane," Journal of Acoustical Society of America, vol. 36, no. 6, pp. 1154-1161, 1964.

[7] B. E. Treeby, J. Jaros, D. Rohrbach, B. T. Cox , "Modelling elastic wave propagation using the k-Wave MATLAB toolbox," IEEE International Ultrasonics Symposium, pp. 146-149, 2014. 